УДК 616-89-008:616-035.1:616-052

Для цитирования: Симуткин Г.Г., Яковлева А.Л. Эффективность фармакотерапии биполярного аффективного расстройства при коморбидности с расстройством личности. Сибирский вестник психиатрии и наркологии. 2017; 3 (96): $97-101$. https://doi.org/10.26617/1810-3111-2017-3(96)-97-101

\title{
Эффективность фармакотерапии биполярного афффективного расстройства при коморбидности с расстройством личности
}

\author{
Симуткин Г.Г., Яковлева А.Л.
}

Научно-исследовательский институт психического здоровья

Томский национальный исследовательский медицинский иеетр Российской академии наук

Россия, 634014, Томск, ул. Алеутская, 4

\section{PEЗЮME}

Цель исследования - сравнительное изучение эффективности проводимой фармакотерапии текущего депрессивного эпизода в случае коморбидности биполярного аффективного расстройства с расстройством личности и в случае биполярного аффективного расстройства без указанной коморбидности. Материал: Основная группа - 20 пациентов с БАР, коморбидным с РЛ. Группа сравнения составила 20 пациентов с диагнозом БАР, но без наличия коморбидного РЛ. Методы: клинико-психопатологический, психометрический (SIGH-SAD, CGI-S, CGI-I, Udvald for Kliniske Undersogelser Scale), статистический. Результаты: результаты исследования показали, что наличие коморбидного РЛ в случае БАР обусловливает большую тяжесть текущего депрессивного эпизода (преимущественно за счет атипичных депрессивных симптомов), чем в случае «чистого» БАР. Чаще возникает необходимость применения комбинированной психофармакотерапии, терапевтический ответ характеризуется более медленной редукцией депрессивных симптомов (также преимущественно за счет атипичных депрессивных симптомов). Регистрируется большая выраженность побочных эффектов от проводимой психофармакотерапии и более медленная их редукция на вторую и четвертую неделю терапии. Заключение. Полученные данные подтверждают необходимость дальнейших исследований для разработки наиболее оптимальных терапевтических подходов в случае коморбидности биполярного аффективного расстройства с расстройством личности.

Ключевые слова: биполярное аффективное расстройство, расстройство личности, коморбидность, комбинированная терапия.

\section{ВВЕДЕНИЕ}

Высокая частота (до 62\%) коморбидности биполярного аффективного расстройства (БАР) и расстройств личности (РЛ) констатируется авторами в ряде исследований [22], однако данные о частоте конкретных РЛ при аффективных расстройствах, в том числе в случае БАР, пока остаются противоречивыми $[19,24]$. В ряде работ указывается на преобладание случаев обсессивно-компульсивного, пограничного и нарциссического РЛ у больных БАР [10]. Из-за перекрытия клинических проявлений БАР и РЛ (особенно пограничного расстройства личности - ПРЛ) ошибочная диагностика может быть смещена в любую сторону, поэтому оценка коморбидности этих расстройств на практике может быть весьма затруднена [20]. Обзор публикаций в библиографической базе данных Medline за 20летний период подтверждает более частую ассоциацию пограничного расстройства личности с БАР, чем с другими психическими расстройствами.

Согласно данным литературы, биполярные пациенты с коморбидным РЛ больше времени проводят в стационаре [9], а также имеют высокий риск суицидального поведения $[11,14,15,22]$. Расстройства личности являются самостоятельной серьезной клинической проблемой [1], а при коморбидности аффективных расстройств с РЛ вполне ожидаемо происходит ухудшение основных клинико-динамических показателей собственно аффективных расстройств и снижение терапевтического ответа.
Современные метааналитические обзоры, посвященные влиянию коморбидности аффективных расстройств и РЛ на основные клинико-динамические характеристики аффективных расстройств (возраст к началу аффективного расстройства, тяжесть аффективной симптоматики, скорость становления ремиссии, частота рецидивов и суицидальное поведение), эффективность медикаментозного и психотерапевтического лечения, а также долгосрочный прогноз и характеристики социальной адаптации у соответствующих пациентов, носят противоречивый характер. С одной стороны, существуют исследования, которые подтверждают факт негативного влияния такого рода коморбидности на вышеуказанные параметры как в случае униполярных, так и биполярных аффективных расстройств [4, $5,6,8,13]$, а также указывают на то, что коморбидные РЛ и отдельные дисфункциональные черты личности снижают эффективность медикаментозного и психотерапевтического лечения аффективных расстройств, создавая трудности рабочего альянса с пациентом и повышая риск преждевременного прерывания терапии [12, 13]. С другой стороны, в ряде работ [16] отмечается, что существенного клинического влияния, в частности на эффективность антидепрессивной терапии, коморбидность аффективных расстройств и РЛ не имеет (особенно при краткосрочной оценке), а в отдельных исследованиях указывается, что личностная патология не является модератором эффекта терапии аффективных расстройств [17]. 
Терапия БАР даже в условиях отсутствия коморбидности с другими психическими расстройствами является сложной клинической задачей [2]. Противоречивость имеющихся литературных данных обусловливает актуальность дальнейшего изучения проблемы клинического значения коморбидности БАР и РЛ.

Цель исследования - сравнительное изучение эффективности проводимой фармакотерапии текущего депрессивного эпизода в случае коморбидности БАР с РЛ и в случае БАР без указанной коморбидности.

\section{МАТЕРИАЛЫ И МЕТОДЫ}

Всего в исследование было включено 40 пациентов (23 женщины в возрасте 44,4 $\pm 10,2$ года и 17 мужчин в возрасте $40,6 \pm 11,0$ года) с текущим умеренным или тяжелым депрессивным эпизодом в рамках БАР в соответствии с критериями МКБ-10 (F31.3, F31.4, F31.5, F31.8) [3]. Общая группа пациентов была разделена на основную группу и группу сравнения. Основная группа - 20 человек (16 женщин - 80\%, 4 мужчин - 20\%) состояла из пациентов с БАР, коморбидным с РЛ. При этом представленность РЛ в основной группе была следующей: в $90 \%$ $(\mathrm{n}=19)$ случаев - смешанное расстройство личности (F61.0), в 5\% (n=1) - пограничное расстройство личности (F60.31), в 5\% (n=1) - истерическое расстройство личности (F60.4). Группа сравнения составила 20 человек (7 женщин - 35\%, 13 мужчин $65 \%$ ) и включала в себя пациентов с диагнозом БАР, но без наличия коморбидного РЛ. Группа сравнения была выделена для оценки эффективности проводимой фармакотерапии текущего депрессивного эпизода у пациентов в случае коморбидности БАР и РЛ и в случае «чистого» (то есть без коморбидности с РЛ) БАР.

Средний возраст пациентов в основной группе на момент обследования был ниже, чем в группе сравнения (соответственно $39,9 \pm 10,8$ года и $46,3 \pm 9,5$ года, p<0,001, t-критерий для независимых групп), что объяснятся более ранним началом БАР при наличии коморбидности с РЛ. По половому составу основная группа и группа сравнения были сопоставимы ( $>>0,05, \chi^{2}$-статистика).

В выделенных группах была проведена сравнительная оценка динамики тяжести депрессии в процессе фармакотерапии (на 0-й, 14-й и 28-й дни) с помощью сокращенной версии шкалы SIGH-SAD (Structured Interview Guide for the Hamilton Depression Rating Scale, Seasonal Affective Disorders Version, Williams J. et al., 1992) [23], включающей в себя 17 пунктов шкалы депрессии Гамильтона и 7 пунктов по оценке атипичных депрессивных симптомов: социальный отход, увеличение веса, увеличение аппетита, гиперфагия, тяга к углеводам, гиперсомния, утомляемость. Кроме того, была проведена оценка тяжести расстройства до начала лечения, на 14-й и 28-й дни фармакотерапии при помощи шкалы CGI-S, а также оценка улучшения психического состояния по шкале CGI-I на 14-й и 28-й дни фармакотерапии. Выраженность побочных эффектов проводимой фармакотерапии оценивалась с помощью шкалы оценки побочного действия - UKU (Udvald for Kliniske Undersogelser Scale) [18].

Статистическая обработка результатов исследования производилась с помощью программы Statistica v.8.0. В случае нормального распределения количественного признака использовался t-критерий Стьюдента. При распределении количественного признака, отличающегося от нормального, применялись критерий Манна-Уитни для независимых групп и критерий Вилкоксона для зависимых групп. Для оценки разницы между ожидаемой и фактической частотой встречаемости признака использовался критерий согласия по К. Пирсону ( $\chi^{2}$-статистика).

\section{РЕЗУЛЬТАТЫ И ОБСУЖДЕНИЕ}

В качестве базисной антидепрессивной терапии пациенты основной группы и группы сравнения получали антидепрессанты из группы селективных ингибиторов обратного захвата серотонина (60\% и 40\%). В отдельных случаях назначались препараты из группы трициклических антидепрессантов, антагонисты/ингибиторы обратного захвата серотонина (тразодон). В целом по частоте назначения различных антидепрессантов при межгрупповом сравнении статистически значимых различий обнаружено не было (p>0,05).

В обеих исследуемых группах часто использовалась комбинированная терапия $(95,0 \%$ и $90,0 \%$, $\mathrm{p}=0,056)$, представленная различными вариантами сочетания психотропных препаратов: антидепрессантов, нормотимиков, нейролептиков, транквилизаторов. При межгрупповом сравнении в основной группе в ходе терапии текущего депрессивного эпизода чаще, чем в группе сравнения, назначалась комбинированная терапия (антидепрессант + нормотимик + нейролептик $(60 \%$ и $40 \%, \mathrm{p}=0,21)$. Применение данного сочетания препаратов в основной группе способствовало снижению уровня эмоциональной нестабильности, было эффективно в отношении поведенческих реакций, способствовало снижению уровня агрессивности, но было сопряжено с большей выраженностью побочных эффектов, объективизированных с помощью шкалы UKU $(\mathrm{p}=0,004)$.

Результаты психометрической оценки эффективности терапии текущего депрессивного эпизода и динамики выраженности побочных эффектов с помощью шкал SIGH-SAD, CGI-S, CGI-I и UKU в соответствующих группах пациентов представлены в виде медианы и межквартильного размаха (табл. 1).

Статистически значимая редукция степени тяжести текущего депрессивного эпизода (включая типичные и атипичные депрессивные симптомы), оцененная по шкале SIGH-SAD, фиксировалась уже на 2й неделе терапии в обеих группах ( $<<0,05$, критерий Вилкосона), но к 14-му и 28-му дням фармакотерапии в основной группе пациентов наблюдалась более выраженная тяжесть депрессии (за счет большей выраженности атипичных депрессивных симптомов), чем 
в группе сравнения ( $<<0,05)$. Межгрупповая оценка тяжести психических расстройств по шкале CGI-S к 14-му $(\mathrm{p}<0,01)$ и 28 -му $(\mathrm{p}<0,001)$ дням терапии также выявила статистически значимую большую выраженность тяжести заболевания у пациентов основной группы, чем в группе сравнения.

Т а б л и ц а 1

\begin{tabular}{|c|c|c|c|c|c|c|}
\hline \multirow{3}{*}{$\begin{array}{r}\text { Эффективн } \\
\text { и дина } \\
\text { в } \\
\text { Шкала } \\
\end{array}$} & $\begin{array}{l}\text { оость те } \\
\text { амики в } \\
3 \text { исслед }\end{array}$ & $\begin{array}{l}\text { рапии те } \\
\text { ыраженг } \\
\text { уемых г }\end{array}$ & руппах & $\begin{array}{l}\text { обочных } \\
\text { (Ме, Q2 }\end{array}$ & जффект & ОВ \\
\hline & \multicolumn{3}{|c|}{ Основная группа } & \multicolumn{3}{|c|}{ Группа сравнения } \\
\hline & 0-я нед. & 2-я нед. & 4-я нед. & 0-я нед. & 2-я нед. & 4-я нед. \\
\hline $\begin{array}{c}\text { Суммарный } \\
\text { балл SIGH- } \\
\text { SAD }\end{array}$ & $\begin{array}{c}29,5 \\
(19,0- \\
34,5)\end{array}$ & \begin{tabular}{|c|}
17,5 \\
$(14,5-$ \\
$24,0)^{*}$
\end{tabular} & $\begin{array}{c}9,0 \\
(7,0- \\
12,0)^{*}\end{array}$ & \begin{tabular}{|c|}
22,5 \\
$(18,0-$ \\
$27,0)$
\end{tabular} & $\begin{array}{c}16,0 \\
(12,5- \\
18,0)\end{array}$ & $\begin{array}{c}7,5 \\
(6,0- \\
9,0)\end{array}$ \\
\hline $\begin{array}{c}\text { Типичные } \\
\text { депрессивные } \\
\text { симптомы } \\
\text { SIGH-SAD } \\
\end{array}$ & $\begin{array}{c}24,0 \\
(16,0- \\
27,0)\end{array}$ & $\begin{array}{c}13,5 \\
(11,0- \\
20,0)\end{array}$ & $\begin{array}{c}7,0 \\
(5,0- \\
10,0)\end{array}$ & $\begin{array}{c}19,0 \\
(16,0- \\
21,5)\end{array}$ & $\begin{array}{c}13,5 \\
(10,0- \\
15,5)\end{array}$ & $\begin{array}{c}6,0 \\
(4,5- \\
7,0)\end{array}$ \\
\hline $\begin{array}{c}\text { Атипичные } \\
\text { депрессивные } \\
\text { симптомы } \\
\text { SIGH-SAD } \\
\end{array}$ & $\begin{array}{c}4,0 \\
(4,0- \\
7,0)^{* *}\end{array}$ & $\begin{array}{c}4,0 \\
(3,5- \\
4,0)^{* *}\end{array}$ & $\begin{array}{c}3,0 \\
(2,0-4,0)\end{array}$ & $\begin{array}{c}2,5 \\
(2,0-4,0)\end{array}$ & $\begin{array}{c}2,0 \\
(1,0-3,5)\end{array}$ & $\begin{array}{c}2,0 \\
(1,0-2,5)\end{array}$ \\
\hline CGI-S & $\begin{array}{c}4,0 \\
(4,0-4,0) \\
\end{array}$ & \begin{tabular}{|c|}
$4,0(4,0-$ \\
$4,0) * *$ \\
\end{tabular} & $\begin{array}{l}3,5(3,0- \\
4,0)^{* * * *}\end{array}$ & $\begin{array}{c}4,0 \\
(4,0-4,0)\end{array}$ & $\begin{array}{c}3,0 \\
(3,0-4,0) \\
\end{array}$ & $\begin{array}{c}2,0 \\
(2,0-, 0) \\
\end{array}$ \\
\hline CGI-I & & $\begin{array}{c}4,0(3,0- \\
4,0)^{* *}\end{array}$ & $\begin{array}{c}3,0(2,0- \\
3,0)^{* * *}\end{array}$ & & $\begin{array}{c}3,0 \\
(2,0-3,0)\end{array}$ & $\begin{array}{c}2,0 \\
(1,0-2,0)\end{array}$ \\
\hline UKU & & $\begin{array}{c}1,0(0,5- \\
2,0)^{*}\end{array}$ & $\begin{array}{c}1,0(0,0- \\
1,5)^{* *}\end{array}$ & $\begin{array}{c}1,0 \\
(0,0-2,0)\end{array}$ & $\begin{array}{c}0,5 \\
(0,0-1,0)\end{array}$ & $\begin{array}{c}0,2 \\
(0,0-0,6)\end{array}$ \\
\hline
\end{tabular}

Пр и м е ч ан и е. Достоверность различий: * - p $<0,05$; $* *-\mathrm{p}<0,01 ; * * *-\mathrm{p}<0,001-$ межгрупповое сравнение.

Оценка степени улучшения психического состояния в исследуемых группах пациентов по шкале CGI-I к 14-му и 28-му дням фармакотерапии выявила, что степень улучшения психического состояния в ходе проводимой терапии была хуже в основной группе, чем в группе сравнения ( $<<0,01)$. Кроме того, в группе пациентов с наличием коморбидности БАР и РЛ отмечалась статистически значимая $(\mathrm{p}<0,05, \mathrm{p}<0,01)$ большая выраженность побочных эффектов в ходе проводимой терапии и меньшая скорость их редукции, чем в группе сравнения.

\section{ЗАКЮЧЕНИЕ}

Таким образом, результаты исследования эффективности фармакотерапии показали, что наличие коморбидного РЛ в случае БАР обусловливает большую тяжесть текущего депрессивного эпизода (преимущественно за счет так называемых атипичных депрессивных симптомов), чем в случае «чистого» БАР. Чаще возникает необходимость применения комбинированной психофармакотерапии, терапевтический ответ характеризуется более медленной редукцией депрессивных симптомов (также преимущественно за счет атипичных депрессивных симптомов). Регистрируется большая выраженность побочных эффектов от проводимой психофармакотерапии и более медленная их редукция на вторую и четвертую недели антидепрессивной терапии. Полученные данные подтверждают необходимость дальнейших исследований для разработки наиболее оптимальных терапевтических подходов в случае коморбидности БАР и РЛ.

\section{КОНФЛИКТ ИНТЕРЕСОВ}

Авторы заявляют об отсутствии конфликта интересов в связи с публикацией данной статьи.

\section{ИСТОЧНИК ФИНАНСИРОВАНИЯ}

Исследование проведено по теме, выполняемой в рамках поисковых научных исследований НИИ психического здоровья «Разработка предикторов прогноза и методов комплексной реабилитации у больных аффективными расстройствами». Шифр темы 0550-2016-0402. Номер госрегистрации АААА-А 16-116042610054-4.

\section{СООТВЕТСТВИЕ ПРИНЦИПАМ ЭТИКИ}

Работа соответствует этическим стандартам, разработанным в соответствии с Хельсинской декларацией ВМА (протокол заседания локального этического комитета при НИИ психического здоровья № 85 от 14 декабря 2015 г., дело № 85/4.2015).

\section{ЛИТЕРАТУРА}

1. Бохан Н.А., Аксенов М.М., Белокрылова М.Ф., Ветлугина Т.П., Никитина В.Б., Гычев А.В., Куприянова И.Е., Рудницкий В.А., Перчаткина О.Э., Смирнова Л.П., Лукьянова Е.В., Дашиева Б.А., Карауш И.С. Клинико-биологические закономерности и социально-психологические предпосылки формирования непсихотических психических расстройств у лиц, проживающих в регионе Сибири. Сибирский вестник психиатрии и наркологии. 2015; 3 (88): С. 9-18.

2. Мосолов С.Н. Биполярное аффективное расстройство: диагностика и терапия. М.: МЕДпресс-информ, 2008: 384.

3. Психические расстройства и расстройства поведения, класс V МКБ-10, адаптированный для использования в Российской Федерации / под общей редакцией Б.А. Казаковцева, В.Б. Голланда. М.: Минздрав России, 1998: 512.

4. Симуткин Г.Г., Яковлева А.Л., Бохан Н.А. Проблема коморбидности аффективных расстройств и расстройств личности (обзор зарубежной литературы). Социальная и клиническая психиатрия. 2014; 24 (2): 91-97.

5. Степанов И.Л., Ваксман А.В. Особенности течения циркулярных депрессий у больных с сопутствующим личностным расстройством. Социальная и клиническая психиатрия. 2013; 23 (4): 32-36.

6. Яковлева А.Л., Симуткин Г.Г. Клинико-динамические особенности аффективных расстройств при их коморбидности с личностными расстройствами. Сибирский вестник психиатрии и наркологии. 2014; 2 (83): 26-29.

7. Bagby R.M., Psych C., Quilty L.C., Ryder A.C. Personality and depression. Can. J. Psychiatr. 2008; 53 (1): 14-25.

8. Bajaj P., Tyrer P.Managing mood disorders and comorbid personality disorders. Current Opinions in Psychiatry. 2005; 18 (1): 27-31.

9. Barbato N., Hafner R.J. Comorbidity of bipolar and personality disorder. Aust. NZ. J. Psychiatry. 1998; 32: 276-280.

10. Brieger P., Ehrt U., Marneros A. Frequency of comorbid personality disorders in bipolar and unipolar affective disorders. Source Compr. Psychiatr. 2003; 44 (1): 28-34.

11. Coryell W., Kriener A., Butcher B., Nurnberger J. Risk factors for suicide in bipolar I disorder in two prospectively studied cohorts. J. Affect. Disord. 2016; 190: 1-5.

12. Diguer L., Barber J.P., Luborsky L. Three concomitants: personality disorders, psychiatric severity, and outcome of dynamic psychotherapy of major depression. Am. J. Psychiatry. 1993; 150: 1246-1248.

13. Grilo C. M., Stout R.L., Markowitz J.C. et al. Personality disorders predict relapse after remission from an episode of major depressive disorder: a 6-year prospective study. J. Clin. Psychiatr. 2010; 71 (12): 1629-1635

14. Isometsä E. Suicidal Behaviour in Mood Disorders - Who, When, and Why? Can. J. Psychiatry. 2014; 59 (3): 120-130.

15. Jylhä P., Rosenström T., Mantere O. et al. Personality disorders and suicide attempts in unipolar and bipolar mood disorders. $J$. Affective Disord. 2016; 190: 632-639. 
16. Kool S., Schoevers R., de Maat S. et al. Efficacy of pharmacotherapy in depressed patients with and without personality disorders: a systematic review and meta-analysis. J. Affect. Disord. 2005; 88: 269-278.

17. Levenson J.C., Wallace M.L., Fournier J.C., Rucci P., Frank E. The role of personality pathology in depression treatment outcome with psychotherapy and pharmacotherapy. J. Consult. Clin. Psychol. 2012; 80 (5): 719-729.

18. Lingjærde O., Ahlfors U.G., Bech P., Dencker S..J., Elgen K. The UKU side effect rating scale. A new comprehensive rating scale for psychotropic drugs and a cross-sectional study of side effects in neuroleptic-treated patients. Acta Psychatr. Scand. 1987; 334: 1100.

19. Mulder R.T. Personality pathology and treatment outcome in major depression: a review. Am. J. Psychiatr. 2002; 159: 359-371.

20. Ruggero C. J., Zimmerman M., Chelminski I. et al. Borderline personality disorder and the misdiagnosis of bipolar disorder. J. Psychiatr. Res. 2010; 44: 405-408.
21. Tamam L., Ozpoyraz N., Karatas G. Personality disorder comorbidity among patients with bipolar I disorder in remission. Acta Neuropsychiatr. 2004; 16: 175-180.

22. Vieta E., Colom F., Martinez-Aran A. et al. Bipolar II disorder and comorbidity. Compr. Psychiatr. 2000; 41: 339-343.

23. Williams J., Link M., Rosenthal N.E. et al. Structured Interview Guide for the Hamilton Depression Rating Scale - Seasonal Affective Disorder Version (SIGH-SAD). New York: New York Psychiatric Institute, 1992.

24. Wongpakaran N., Boonyanaruthee V., Pinyopornpanish M. Comorbid personality disorders among patients with depression. Neuropsychiatric Disease and Treatment. 2015; 11: 1091-1096.

Поступила в редакцию 11.05.2017 Утверждена к печати 26.06.2017

Симуткин Герман Геннадьевич, д.м.н., в.н.с. отделения аффективных состояний.

Яковлева Альбина Леонтьевна, к.м.н., врач-психиатр третьего клинического психиатрического отделения.

Симуткин Герман Геннадьевич, ggsimutkin@gmail.com

УДК 616-89-008:616-035.1:616-052

For citation: Simutkin G.G., Yakovleva A.L. Efficiency of pharmacotherapy of bipolar affective disorder comorbid with personality disorder. Siberian Herald of Psychiatry and Addiction Psychiatry. 2017; 3 (96): 97-101. https://doi.org/10.26617/1810-31112017-3(96)-97-101

\section{Efficiency of pharmacotherapy of bipolar affective disorder comorbid with personality disorder}

\section{Simutkin G.G., Yakovleva A.L.}

Mental Health Research Institute, Tomsk National Research Medical Center, Russian Academy of Sciences Aleutskaya Street 4, 634014, Tomsk, Russian Federation

\section{ABSTRACT}

Objective: the comparative study of the efficiency of conducted pharmacotherapy of the current depressive episode in the case of comorbidity of bipolar affective disorder (BAD) with personality disorder (PD) and in the case of bipolar affective disorder without such comorbidity. Material: The main group - 20 patients suffering from bipolar affective disorder with personality disorder. The group of comparison included 20 patients with the diagnosis of bipolar affective disorder, but without presence of comorbid PD. Methods: clinical-psychopathological, psychometric (SIGH-SAD, CGI-S, CGI-I, Udvald for Kliniske Undersogelser Scale), statistical. Results: results of the study showed that presence of comorbid PD in the case of BAD caused major severity of the current depressive episode (predominantly due to atypical depressive symptoms) than in the case of "pure" BAD. The need of applying combined psychopharmacotherapy appeared more frequently, therapeutic response was characterized by a slower reduction of depressive symptoms (also predominantly due to atypical depressive symptoms). Major severity of side effects from conducted psychopharmacotherapy and their slower reduction by weeks 2 and 4 of the therapy were documented. Conclusion. The findings confirmed the need of further studies for developing the most optimal therapeutic approaches in the case of comorbidity of bipolar affective disorder with personality disorder.

\section{Keywords: psychopharmacotherapy, antidepressants, biological rhythms.}

\section{REFERENCES}

1. Bokhan N.A., Axenov M.M., Belokrylova M.F., Vetlugina T.P., Nikitina V.B., Gychev A.V., Kupriyanova I.E., Rudnitsky V.A. Perchatkina O.E., Smirnova L.P., Lukiyanova E.V., Dashieva B.A., Karaush I.S. Kliniko-biologicheskie zakonomernosti i sotsial'no-psikhologicheskie predposylki formirovaniya nepsikhoticheskikh psikhicheskikh rasstroystv u lits, prozhivayushchikh v regione Sibiri [Clinical-biological regularities and socialpsychological preconditions of formation of non-psychotic mental disorders in persons residing in region of Siberia]. Sibirskiy vestnik psikhiatrii i narkologii - Siberian Herald of Psychiatry and Addiction Psychiatry. 2015; 3 (88): C. 9-18 (in Russian).

2. Mosolov S.N. Bipolyarnoe affektivnoe rasstroystvo: diagnostika terapiya [Bipolar affective disorder: diagnosis and therapy]. M.: MEDpress-inform, 2008: 384 (in Russian).
3. Psikhicheskie rasstroystva i rasstroystva povedeniya, klass $\mathrm{V}$ MKB-10, adaptirovannyy dlya ispol'zovaniya v Rossiyskoy Federatsii [Mental disorders and behavioral disorders, class V of ICD10, adjusted for use in the Russian Federation]. B.A. Kazakovtsev, V.B. Holland, eds. M.: Ministry of Healthcare of the Russian Federation, 1998: 512 (in Russian).

4. Simutkin G.G., Yakovleva A.L., Bokhan N.A. Problema komorbidnosti affektivnykh rasstroystv i rasstroystv lichnosti (obzor zarubezhnoy literatury) [Comorbidity of affective and personality disorders]. Sotsial'naya i klinicheskaya psikhiatriya - Social and Clinical Psychiatry. 2014; 24 (2): 91-97 (in Russian).

5. Stepanov I.L., Vaxman A.V. Osobennosti techeniya tsirkulyarnykh depressiy u bol'nykh s soputstvuyushchim lichnostnym rasstroystvom [The course of cyclical depressions in patients with comorbid personality disorders]. ]. Sotsial'naya i klinicheskaya psikhiatriya - Social and Clinical Psychiatry. 2013; 23 (4): 32-36 (in 
Russian).

6. Yakovleva A.L., Simutkin G.G. Kliniko-dinamicheskie osobennosti affektivnykh rasstroystv pri ikh komorbidnosti s lichnostnymi rasstroystvami [Clinical-dynamic features of affective disorders in their comorbidity with personality disorders]. Sibirskiy vestnik psikhiatrii i narkologii - Siberian Herald of Psychiatry and Addiction Psychiatry. 2014; 2 (83): 26-29 (in Russian).

7. Bagby R.M., Psych C., Quilty L.C., Ryder A.C. Personality and depression. Can. J. Psychiatr. 2008; 53 (1): 14-25.

8. Bajaj P., Tyrer P. Managing mood disorders and comorbid personality disorders. Current Opinions in Psychiatry. 2005; 18 (1): 27-31.

9. Barbato N., Hafner R.J. Comorbidity of bipolar and personality disorder. Aust. NZ. J. Psychiatry. 1998; 32: 276-280.

10. Brieger P., Ehrt U., Marneros A. Frequency of comorbid personality disorders in bipolar and unipolar affective disorders. Source Compr. Psychiatr. 2003; 44 (1): 28-34.

11. Coryell W., Kriener A., Butcher B., Nurnberger J. Risk factors for suicide in bipolar I disorder in two prospectively studied cohorts. J. Affect. Disord. 2016; 190: 1-5.

12. Diguer L., Barber J.P., Luborsky L. Three concomitants: personality disorders, psychiatric severity, and outcome of dynamic psychotherapy of major depression. Am. J. Psychiatry. 1993; 150: 1246-1248.

13. Grilo C. M., Stout R.L., Markowitz J.C. et al. Personality disorders predict relapse after remission from an episode of major depressive disorder: a 6-year prospective study. J. Clin. Psychiatr. 2010; 71 (12): 1629-1635.

14. Isometsä E. Suicidal Behaviour in Mood Disorders - Who, When, and Why? Can. J. Psychiatry. 2014; 59 (3): 120-130.

15. Jylhä P., Rosenström T., Mantere O. et al. Personality disorders and suicide attempts in unipolar and bipolar mood disorders. $J$. Affective Disord. 2016; 190: 632-639.

16. Kool S., Schoevers R., de Maat S. et al. Efficacy of pharmacotherapy in depressed patients with and without personality disorders: a systematic review and meta-analysis. J. Affect. Disord. 2005; 88: 269-278.

17. Levenson J.C., Wallace M.L., Fournier J.C., Rucci P., Frank E. The role of personality pathology in depression treatment outcome with psychotherapy and pharmacotherapy. J. Consult. Clin. Psychol. 2012; 80 (5): 719-729.

18. Lingjærde O., Ahlfors U.G., Bech P., Dencker S..J., Elgen K. The UKU side effect rating scale. A new comprehensive rating scale for psychotropic drugs and a cross-sectional study of side effects in neuroleptic-treated patients. Acta Psychatr. Scand. 1987; 334: 1100.

19. Mulder R.T. Personality pathology and treatment outcome in major depression: a review. Am. J. Psychiatr. 2002; 159: 359-371.

20. Ruggero C. J., Zimmerman M., Chelminski I. et al. Borderline personality disorder and the misdiagnosis of bipolar disorder. J. Psychiatr. Res. 2010; 44: 405-408.

21. Tamam L., Ozpoyraz N., Karatas G. Personality disorder comorbidity among patients with bipolar I disorder in remission. Acta Neuropsychiatr. 2004; 16: 175-180.

22. Vieta E., Colom F., Martinez-Aran A. et al. Bipolar II disorder and comorbidity. Compr. Psychiatr. 2000; 41: 339-343.

23. Williams J., Link M., Rosenthal N.E. et al. Structured Interview Guide for the Hamilton Depression Rating Scale - Seasonal Affective Disorder Version (SIGH-SAD). New York: New York Psychiatric Institute, 1992.

24. Wongpakaran N., Boonyanaruthee V., Pinyopornpanish M. Comorbid personality disorders among patients with depression. Neuropsychiatric Disease and Treatment. 2015; 11: 1091-1096.

Поступила в редакцию 11.05.2017

Simutkin German G., MD, leading researcher of Affective States Department, Mental Health Research Institute, Tomsk National Research Medical Center, Russian Academy of Sciences, Tomsk, Russian Federation.

Yakovleva Al'bina L., PhD, psychiatrist of the Third Clinical Psychiatric Unit, Mental Health Research Institute, Tomsk National Research Medical Center, Russian Academy of Sciences, Tomsk, Russian Federation.

Simutkin German G., ggsimutkin@gmail.com 